\title{
Experimental analysis of artificial dragonfly wings using black graphite and fiberglass for use in Biomimetic Micro Air Vehicles (BMAVs)
}

\author{
Praveena Nair ${ }^{1, a}$, Thomas Arthur Ward ${ }^{2, b}$, Rubentheren Viyapuri ${ }^{3, c}$, and Mohd \\ Rafie Johan ${ }^{4, d}$ \\ 1,2,3,4 Department of Mechanical Engineering, Faculty of Engineering, University of Malaya,50603, Kuala Lumpur, Malaysia
}

\begin{abstract}
This article examines the suitability of two different materials which are black graphite carbon fiber and red pre-impregnated fiberglass from which to fabricate artificial dragonfly wing frames. These wings could be of use in Biomimetic Micro Aerial Vehicles (BMAV). BMAV are a new class of unmanned micro-sized air vehicles that mimic flying biological organisms. Insects, such as dragonflies, possess corrugated and complex vein structures that are difficult to mimic. Simplified dragonfly wing frames were fabricated from these materials and then a nanocomposite film was adhered to them, which mimics the membrane of an actual dragonfly. Experimental analysis of these results showed that although black graphite carbon fiber and red pre-impregnated fiberglass offer some structural advantages, red pre-impregnated fiberglass was a less preferred option due to its warpage and shrinking effects. Black graphite carbon fiber with its high load bearing capability is a more suitable choice for consideration in future BMAV applications.
\end{abstract}

\section{Introduction}

Biomimetic micro air vehicle (BMAV) is a micro-scaled aircraft that mimics the flapping wing motion of insects or small birds (e.g.: dragonfly and hummingbirds). Dragonflies are powerful and agile fliers, and its wings exhibit excellent functional characteristics through the coupling and synergy of configuration, structure and material. At least four distinct flight styles are recognized in Odonata which is counter-stroking (where the forewings and hindwings move up and down about 180 degrees), phased-stroking (where the hindwings cycle about 90 degrees before the forewings), synchronized stroking where the forewing and hindwings move together in unison, and gliding. Dragonfly wings are dynamic, non-planar structures. The corrugations in the wing hold an aero foil of air around it which lowers friction and the wings rotate around several axes, responding both to muscle actions and to inertia effects. The structural characteristics possessed by dragonfly wings provide a biological inspiration for the investigation and development of flapping-wing micro air vehicles. The US Defense Advanced Research Projects Agency (DARPA) released a Broad Agency Announcement (BAA 97-29) in 1997, defining micro air vehicles to be less than $15 \mathrm{~cm}$ in any dimension. Later in

\footnotetext{
${ }^{\mathrm{a}}$ Corresponding author: ss.praveenanair@hotmail.com

bdrtomward@um.edu.my,

crubentheren@live.com,

dmrafiej@um.edu.my
}

2005, DARPA defined nano air vehicles (BAA 06-06) as being no larger than $7.5 \mathrm{~cm}$ or heavier than $10 \mathrm{~g}$ (carrying a $2 \mathrm{~g}$ payload). Their miniature size makes them difficult to detect, easy to be deployed by a single operator, inexpensive to fabricate and allows the potential to fly inside buildings or compact spaces. BMAVs are envisioned for use on civil and military missions that are of a limited duration, such as: remote sensing of hazard sites (e.g. chemical spill, radiation, high voltage power lines etc.), indoor video mapping, and police or military surveillance. The artificial wings ofBMAV must be flexible and strong enough to endure the various forces produced by flapping motion just like an actual insect. The major form of deformation that the wings undergo during flight is bending and twisting, and these wings will have to be able to undergo the forces that act on its surface. Very few fabrication methods for small insect artificial wings have been proposed. Pornsin-sirirak et al presented the first microelectromechanical systems (MEMS) photolithography and etching method, in which the vein and membrane of the wing are fabricated using titanium alloy (Grade 5) and parylene-C, respectively [1]. Nguyen et al created artificial wings that mimic the main venation structure of a beetle using pre-impregnated carbon/epoxy fiber for the framing structure and thin Kapton film for the membrane [8]. Bao et al 
describes the design and micromachining of a threedimensional BMAV wing from a stainless steel (SU-8) material using microelectromechanical system (MEMS) lithography [2]. Very little has been written about the BMAV wing frame structures that encase the membrane.In this article we describe a novel methodology of fabricating BMAV wings (based on the DiplacodesBipunctata dragonfly) using two different materials as the frame structure which is black graphite carbon fiber, and red prepreg fiberglass. The structures were fabricated after designing a simplified structure of the dragonfly. Dragonfly wings are highly corrugated with complex vein structures. A simplified model of this wing was created using spatial network analysis taking the pattern density into account. This is described in detail in another article written by the authors of this work [6].Wing membrane was formed by immersing the structures in a chitosan nano-composite suspension, with chitin whiskers as a physical reinforcement and tannic acid as the crosslinking agent, using the casting evaporation method [7]. After 48 hours of solidification a nano-composite film with a thickness of

$0.03 \mathrm{~mm}$ was created. Tensile and bending test measurements were conducted on these frame structures and the results were observed.

\section{Methodology}

\subsection{Wing structure overview}

Fig. 1 shows a comparison between the detailed vein structure models of the dragonfly wings and their corresponding simplified vein structure models. As Figure 1 illustrates dragonflies have different forewing and hindwing geometries, so both were considered. As previously described, the simplified models were created using spatial network analysis [6]. This method utilizes the pattern density of the membrane and has been widely used in Geographical InformationSystems (GIS) to explore spatial patterns.

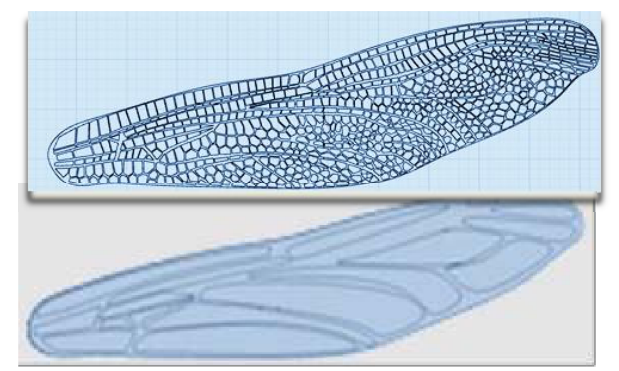

Figure 1.The simplified model of a dragonfly wing. a) detailed model of forewing, b) simplified model of forewing.

A spatial network (sometimes also geometric graph) is a graph in which the vertices or edges are spatial elements associated with geometric objects, which means the nodes are located in a space within a specified unit (e.g. radius or distance). The mathematical simplest realization is a random geometric graph where nodes are distributed uniformly at random onto a two-dimensional plane [8]. There are various methods of conducting spatial network analysis. Geographical Information Systems (GIS) used this method to explore geographic spatial patterns. Our methodology is similar, but we are applying this algorithm (based on shape, angle and density) to a biological structure (a dragonfly wing). The idea of simplifying a dragonfly wing based on spatial network analysis was inspired by observing its compactly arranged geometrical patterns. Although there are a variety of methods available to conduct a spatial network analysis, the proximity proposition method was chosen for this analysis because of its conformity of use to a wing structure. A circle is the most compact geometric shape possible [9], so this was selected as a reference defining the specified unit (radius). The proximity proposition is defined as the shortest average distance of a circle in a given area of symmetrical shapes. The proximity index is the ratio of the proximate center from all points in the shape. Proximity index can be calculated by (1)

$$
d_{c s}=\left(\frac{1}{n}\right) \sum_{i=1}^{n}\left(x_{i}-x_{c}\right)^{2}+\left(y_{i}-y_{c}\right)^{2}
$$

\subsection{Materials of frame structure}

Several geometrically identical wings were fabricated based on the two simplified models. Although the same nano-composite was used for each wing membrane, the frames were fabricated from two different materials: black graphite carbon fiber laminates and red preimpregnated fiberglass laminates. Three samples of each wing type were tested to determine their tensile strength performance. The compromise between minimizing the weight and maintaining adequate tensile strengths are critical for BMAV wings.

\subsection{Fabrication of black graphite and red fiber wings}

Black graphite carbon fiber sheets were purchased and visually inspected to make certain they were uniform in appearance and free from foreign material. Each carbon fiber laminate is $4 \mathrm{~mm}$ thick. The woven laminate was hardened using an epoxy resin with a hardener (Araldite rapid kit). Both epoxy resin and hardener were mixed with a ratio of 1:1 and then applied evenly to the laminate in a thin layer. The laminate was then left to cure in a Memmert UNB 300 convection oven for about two hours at a constant temperature of $45^{\circ} \mathrm{C}$. The forewing and hindwing shapes were then carved out of the hardened laminates manually with a knife. Red pre-impregnated fiberglass plies with a thickness of $0.03 \mathrm{~mm}$ were purchased and visually inspected. After curing, the red pre-impregnated fiberglass exhibits a plastic-like characteristic. Unlike carbon fiber which will retain its strand-like properties. This increases the chance of delamination if a resin and hardener is not applied. The red pre-impregnated fiberglass (after curing) does not require an extra application of resin or hardener. In order to create the wing frame structure, the material was warmed as necessary to enable easier manual carving with a knife. Fig 2.shows the finished wings. 


\subsection{Chitosan nano-composite solution}

The chitosan nano-composite suspension as mentioned earlier was made up of a chitosan suspension reinforced with nano-sized chitin whiskers and crosslinked using tannic acid. This nano-composite film was processed by our research team for this specific purpose and is featured in another article [7]. The suspension was transformed into a thin $3 \mathrm{~mm}$ film by the casting evaporation method. This film was chosen because it closely mimics the material properties of a dragonfly wing membrane. The mechanical properties and water resistivity of chitosan film can be controlled by the addition of chitin whiskers and tannic acid as a crosslinker.Once cured, the film created a shiny, transparent film layer that adheres firmly to the frame structure.
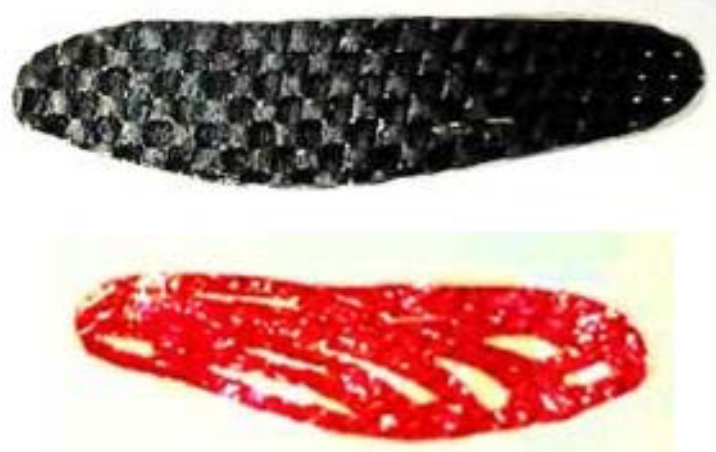

Figure 2.Fabricated wings.

\subsection{Experimental set up}

Wing frame models were subjected to tensile testing using the Instron Universal Testing Machine (Model 5569). The maximum load capacity for this machine is 50 $\mathrm{kN}$. The data was compiled by Instron MERLIN software. A specific jig was designed for tensile test to clamp the fabricated models, whereby the slots were used to clamp the thin structures firmly. All testing was repeated three times for each wing type, necessitating the fabrication of three identical copies of each wing type. Average measurements of the three tests of identical wings are shown in the figures located in Sections 3.3 and 3.4. (The standard deviations of all these tests are less than $2 \%$, which was too small to plot as error bars on the figures.) Since the wing frame test specimen is a sheet-like structure the same ratio of width-to-gage length (18:18 for forewing and 20:20 for hindwing) was maintained in order to compensate for the elongation that occurs during diffuse necking. ASTM test specifications were followed throughout the experiment. Each base of the wing models were clamped at a fixed point and the tips were clamped at the moving point. The load was then increased gradually with a speed rate of $0.013 \mathrm{~m} / \mathrm{s}$. The rate of extension was observed until the wing model experienced a structural failure. This point can be observed in the collected data as a sudden decrease in the load. Data obtained in RAW file was then converted to Microsoft Excel for further analysis.

\section{Results and Discussion}

\subsection{Tensile test experimental results}

Figures 3 and 4 compare the forewing for two different frame structure materials. They also compare the results of models with and without membranes, allowing the effects of the chitosan film on the frame structures to be observed. The two materials (without membrane) exhibit their original properties under tensile stress, which agrees with previous studies. Both figures show a sudden decline in tensile stress, which indicates failure (fracture) has occurred (destructive testing). Figure 3 shows a comparison of carbon fiber wings, with and without film membranes. The adhesion of chitosan nano-composite film yields an immense increase in strength. The peak tensile strength for the forewings, with and without membranes, are 48.07 and $1.32 \mathrm{MPa}$, respectively. The results for the hindwings, with and without membranes, are 40.06 and $1.34 \mathrm{MPa}$, respectively. This shows that the addition of film increases the ultimate strength by $2976 \%$ to $3592 \%$. This shows that the adhesion of the film to these carbon fiber models greatly intensifies its ultimate strength.Figure 4 shows the results obtained for red preimpregnated fiberglass model wings. These results also show an increase in ultimate strength after adhesion of the chitosan nano-composite film. The ultimate strength for the forewing, with and without membranes, are: 2.03 and $0.01 \mathrm{MPa}$, respectively. The ultimate strength for the hindwings, with and without membranes, are 3.04 and $0.01 \mathrm{MPa}$, respectively. However, the red fiberglass undergoes warping and shrinkage at a very early stage of the experiment ( 1 minute after starting the experiment). So this phenomenon must be taken under consideration when considering this material for fabrication purpose. Referring to Figures 3 and 4, the percent differences between the simulation and experiments are approximately $5.75 \%$ and $8.93 \%$, with and without membranes, respectively.
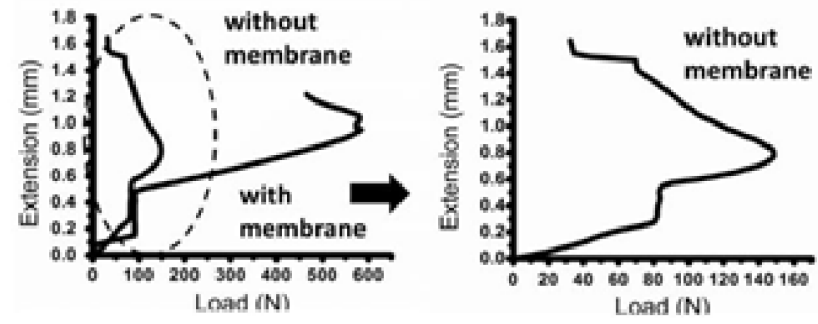

Figure 3.Load vs Extension curves for carbon fiber forewing

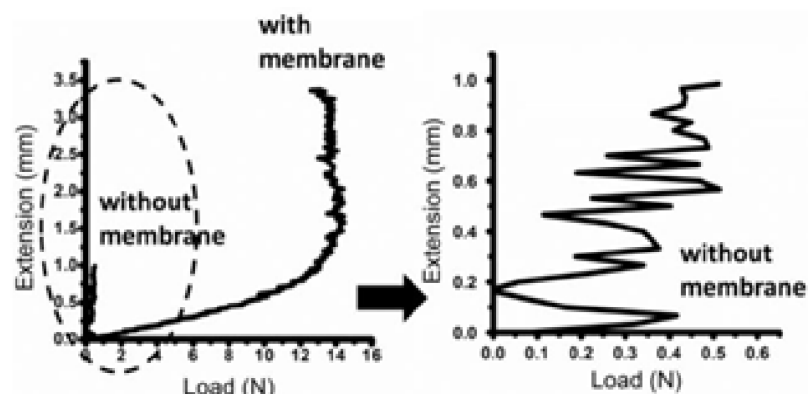

Figure 4. Load vs Extension curves for red pre-impregnated fiberglass forewing 


\section{Conclusion}

Results obtained shows that the black graphite carbon fiber has excellent load bearing capabilities and could be considered for future use to fabricate BMAVS. Red fiber could be considered as a potential material due to its light weight but the warpage and shrinkage proves to be a major disadvantage.

\section{Acknowledgements}

This research was done under the auspices of the Centre for Transportation Research at the Faculty of Engineering, University of Malaya. It is primarily funded by High Impact Research Grant UM.C/625/1/HIR/MOHE/ENG/53 (H-16001-D000053) along with a secondary grant from the University of Malaya: RG155-12AET.

\section{References}

[1] T. N. Pornsin-sirirak, Y. C. Tai, H. Nassef, and C. M. Ho, "Titanium-alloy MEMS wing technology for a micro aerial vehicle application," Sensors and Actuators A: Physical, vol. 89, pp.95-103, 3/20/ (2001).

[2] X. Q. Bao, T. Dargent, S. Grondel, J. B. Paquet, and E. Cattan, "Improved micromachining of all SU-8 3D structures for a biologically-inspired flying robot," Microelectronic Engineering, vol.88, pp. 2218-2224, 8// (2011).

[3] Q.-T. Truong, B. W. Argyoganendro, and H. C. Park, "Design and Demonstration of Insect Mimicking Foldable Artificial Wing Using Four-Bar Linkage
Systems," Journal of Bionic Engineering, vol. 11, pp. 449-458, 7// (2014).

[4] M. S. David Kumar, PM Mohite, SudhirKamle, "Structural Dynamic Analysis of BioInspired Carbon Fiber/Polythene MAV Wings," presented at the Recent Advances in Mechanical Engineering, India, (2014).

[5] J. H. K.,N. Seo and G. a. D. Byun, "Micro/nanofabrication for a realistic beetle wing with a superhydrophobic surface," Bioinspiration and Biomimetics, vol. 7, pp. 1748-3190, (2012).

[6] Praveena N. Sivasankaran, Thomas A. Ward, "Spatial Network Analysis to Construct SimplifiedWing Structural Models for Biomimetic MicroAir Vehicles"," unpublished|.

[7] V. Rubentheren, Thomas. A. Ward, C. Y. Chee, and C. K. Tang, "Processing and analysis of chitosan nanocomposites reinforced with chitin whiskers and tannic acid as a crosslinker," Carbohydrate Polymers, vol. 115, pp. 379-387, 1/22/ (2015).

[8] D. Akın, A. Kasgoz, and A. Durmus, "Quantifying microstructure, electrical and mechanical properties of carbon fiber and expanded graphite filled cyclic olefin copolymer composites," Composites Part A: Applied Science and Manufacturing, vol. 60, pp. 44-51, 5// (2014).

[9] Boeing, "Boeing Material Specification," in Glass fabric reinforcements for laminated plastic products, ed, (2000).

[10] Boeing, "Boeing Material Specification," in Glass fabric preimpregnated epoxy resin low temperature curing, ed, (2003).

[11] Boeing, "Boeing Material Specification" in Carbon fiber reinforcements, yarn and fabric vol.BMS 8-9J, ed, (2004) 